\title{
Profiling and initial validation of urinary microRNAs as biomarkers in IgA nephropathy
}

Nannan Wang, Ru Bu, Zhiyu Duan, Xueguang Zhang, Pu Chen, Zuoxiang Li, Jie Wu, Guangyan Cai, Xiangmei Chen

Background: MicroRNAs (miRNAs) have been found in virtually all body fluids and used successfully as biomarkers for various diseases. Evidence indicates that miRNAs have important roles in IgA nephropathy (IgAN), a major cause of renal failure. In this study, we looked for differentially expressed miRNAs in IgAN and further evaluated the correlations between candidate miRNAs and the severity of IgAN.

Methods: Microarray and RT-qRCR (real-time quantitative polymerase chain reaction) were sequentially used to screen and further verify miRNA expression profiles in urinary sediments of IgAN patients in two independent cohorts. The screening cohort consisted of 32 urine samples from 18 patients with IgAN, 4 patients with MN (membranous nephropathy), 4 patients with MCD (minimal changes disease) and 6 healthy subjects; the validation cohort consisted of 102 IgAN patients, 41 MN patients, 27 MCD patients and 34 healthy subjects. The renal pathological lesions of patients with IgAN were evaluated according to Lee's grading system and Oxford classification.

Results: At the screening phase, significance analysis of microarrays analysis showed that no miRNA was differentially expressed in the IgAN group compared to all control groups. But IgAN grade I-II and III subgroups (according to Lee's grading system) shared dysregulation of two miRNAs (miR-3613-3p and miR-4668-5p). At the validation phase, RTqPCR results showed that urinary level of miR-3613-3p was significantly lower in IgAN than that in MN, MCD and healthy controls $(0.47,0.44$ and 0.24 folds, respectively, all $P<0.01$ by Mann-Whitney $U$ test); urinary level of miR-4668-5p was also significantly lower in IgAN than that in healthy controls $(0.49$ fold, $P<0.01)$. Significant correlations were found between urinary levels of miR-3613-3p with 24-hour urinary protein excretion (Spearman $r$ $=0.50, P=0.034)$, eGFR (estimated glomerular filtration rate) $(r=-0.48, P=0.043)$ and Lee's grades $(r=0.57, P=0.014)$. Similarly, $m i R-4668-5 p$ was significantly correlated with eGFR ( $r=-0.50, P=0.034)$ and Lee's grades $(r=0.57, P=0.013)$. For segmental glomerulosclerosis according to Oxford classification, patients scored as SO had significantly lower levels of urinary miR-3613-3p and miR-4668-5p than those scored as S1 
(0.41 and 0.43 folds, respectively, all $P<0.05$ ).

Conclusions: The expression profile of miRNAs was significantly altered in urinary sediments from patients with IgAN. Urinary expression of miR-3613-3p was downregulated in patients with IgAN. Moreover, urinary levels of both miR-3613-3p and miR4668-5 $p$ were correlated with disease severity. Further studies are needed to explore the roles of miR-3613-3p and miR-4668-5p in the pathogenesis and progression of $\lg \mathrm{A}$ nephropathy. 
2 Profiling and initial validation of urinary microRNAs as biomarkers in IgA 3 nephropathy

4 Nannan Wang, Ru Bu, Zhiyu Duan, Xueguang Zhang, Pu Chen, Zuoxiang Li, Jie

5 Wu, Guangyan Cai, Xiangmei Chen

6 Background: MicroRNAs (miRNAs) have been found in virtually all body fluids

7 and used successfully as biomarkers for various diseases. Evidence indicates

8 that miRNAs have important roles in IgA nephropathy (IgAN), a major cause of

9 renal failure. In this study, we looked for differentially expressed miRNAs in IgAN

10 and further evaluated the correlations between candidate miRNAs and the 11 severity of IgAN.

12 Methods: Microarray and RT-qRCR (real-time quantitative polymerase chain

13 reaction) were sequentially used to screen and further verify miRNA expression

14 profiles in urinary sediments of IgAN patients in two independent cohorts. The

15 screening cohort consisted of 32 urine samples from 18 patients with IgAN, 4 16 patients with $\mathrm{MN}$ (membranous nephropathy), 4 patients with MCD (minimal

17 changes disease) and 6 healthy subjects; the validation cohort consisted of 102

18 IgAN patients, $41 \mathrm{MN}$ patients, $27 \mathrm{MCD}$ patients and 34 healthy subjects. The

19 renal pathological lesions of patients with IgAN were evaluated according to Lee's 20 grading system and Oxford classification. 
21 Results: At the screening phase, significance analysis of microarrays analysis

22 showed that no miRNA was differentially expressed in the IgAN group compared

23 to all control groups. But IgAN grade I-II and III subgroups (according to Lee's

24 grading system) shared dysregulation of two miRNAs (miR-3613-3p and miR-

$254668-5 p)$. At the validation phase, RT-qPCR results showed that urinary level of 26 miR-3613-3p was significantly lower in IgAN than that in MN, MCD and healthy 27 controls $(0.47,0.44$ and 0.24 folds, respectively, all $P<0.01$ by Mann-Whitney $U$ 28 test); urinary level of $\operatorname{miR}-4668-5 p$ was also significantly lower in IgAN than that 29 in healthy controls $(0.49$ fold, $P<0.01)$. Significant correlations were found 30 between urinary levels of miR-3613-3p with 24-hour urinary protein excretion 31 (Spearman $r=0.50, P=0.034)$, eGFR (estimated glomerular filtration rate) $(r=-$

$320.48, P=0.043)$ and Lee's grades $(r=0.57, P=0.014)$. Similarly, miR-4668-5p 33 was significantly correlated with eGFR $(r=-0.50, P=0.034)$ and Lee's grades $(r$ $34=0.57, P=0.013)$. For segmental glomerulosclerosis according to Oxford 35 classification, patients scored as S0 had significantly lower levels of urinary miR$36 \quad 3613-3 p$ and $m i R-4668-5 p$ than those scored as $\mathrm{S} 1$ (0.41 and 0.43 folds, 37 respectively, all $P<0.05)$.

38 Conclusions: The expression profile of miRNAs was significantly altered in urinary 39 sediments from patients with IgAN. Urinary expression of miR-3613-3p was 40 down-regulated in patients with IgAN. Moreover, urinary levels of both miR-3613- 
$413 p$ and miR-4668-5p were correlated with disease severity. Further studies are

42 needed to explore the roles of $m i R-3613-3 p$ and $m i R-4668-5 p$ in the pathogenesis

43 and progression of $\lg A$ nephropathy. 
45 Nannan Wang, Ru Bu, Zhiyu Duan, Xueguang Zhang, Pu Chen, Zuoxiang Li, Jie

46 Wu, Guangyan Cai, Xiangmei Chen*

47 Department of Nephrology, Chinese PLA General Hospital, Chinese PLA Institute

48 of Nephrology, State Key Laboratory of Kidney Diseases, National Clinical

49 Research Center for Kidney Diseases, Beijing 100853, China

50 Corresponding author: Xiangmei Chen

51 Address: Department of Nephrology, Chinese PLA General Hospital, State Key

52 Laboratory of Kidney Diseases, 28 Fuxing Road, Beijing 100853, China.

53 Tel: +86 010 66935462; fax: +8601068130297.

54 E-mail addresses: xmchen301@126.com 
Immunoglobulin A nephropathy (IgAN), the most common type of primary glomerulonephritis worldwide, is characterized by a predominant deposition of IgA-containing immune complexes in the glomerular mesangium (Wyatt \& Julian 2013). The diagnosis of IgAN relies entirely on a renal biopsy, which is invasive and cannot be frequently repeated in the same patient. The prognosis of this disease is good in the early stages, but over the next 20 years, up to $40 \%$ of affected patients will develop irreversible end-stage renal disease (ESRD) (Schena 1990). Therefore, the development of non-invasive biomarkers would be of great significance for clinical assessment of IgAN.

In recent years, the role of microRNAs (miRNAs) in a variety of physiological and pathophysiological processes has received much attention. MiRNAs are a class of small non-coding RNAs that regulate gene expression at the post-transcriptional level (Esteller 2011). It has been demonstrated that miRNAs exhibit functional dysregulation in IgAN (Chandrasekaran et al. 2012; Szeto \& Li 2014). As miRNAs are easily accessible, relatively stable and resistant to RNase-mediated degradation in body fluids (Chen et al. 2008), they have the potential to be used as non-invasive biomarkers.

Recent studies have shown that urinary levels of several selected miRNAs were significantly changed in patients with IgAN compared to healthy individuals (Wang et al. 2010; Wang et al. 2011). However, to date, no data are available concerning the global urinary profile of miRNAs in IgAN patients, and a study with both healthy controls and disease controls is lacking. Therefore, the aim of this study is to find differentially expressed miRNAs in urinary sediments from IgAN patients, and further evaluate the correlations between candidate miRNAs and the severity of IgAN.

\section{Methods}

\section{Statement on ethics}

The study was carried out in accordance with the Declaration of Helsinki for Human Research and 
approved by the Ethics Committee of the Chinese PLA General Hospital (approval number S2014-004-02). Written informed consent for inclusion was obtained from each participant.

\section{Study design}

A screening and a validation phase were designed for the present study. Besides healthy controls, we recruited patients with membranous nephropathy $(\mathrm{MN})$ or minimal change disease (MCD) as other glomerulopathy controls. In the screening phase, 32 urine samples collected from 18 patients with IgAN and 14 controls were subjected to Affymetrix GeneChip miRNA 4.0 Array to identify miRNAs that were significantly differentially expressed. In the validation phase, the expression of different miRNAs was validated by real-time quantitative polymerase chain reaction (RT-qPCR) in samples from 102 patients with IgAN, 41 patients with MN, 27 patients with MCD and 34 healthy controls.

\section{Enrollment of subjects}

Patients who received renal biopsy for the first time from December 2013 to November 2014 at the Nephrology Department of the Chinese PLA General Hospital were consecutively recruited for the present study. The diagnosis was confirmed based on clinical findings and renal biopsies. A total of 322 consecutive patients were diagnosed as IgAN, MN or MCD. Among them, we excluded 41 patients because they were < 20 or $>50$ years old at renal biopsy, 26 patients because they had been treated with steroids within 6 months prior to renal biopsy, and 7 patients because there were less than eight glomeruli in light microscopy sections. We also excluded patients with a concomitant diagnosis of chronic hepatic disease $(n=14)$, diabetes $(n=$ 11), urinary, respiratory or gastrointestinal tract infection $(n=7)$, rheumatoid arthritis $(n=3)$, HenochSchonlein purpura $(n=7)$ and systemic lupus erythematosus $(n=10)$.

Healthy volunteers who were between 20 and 50 years old were recruited at the Physical Examination Center of the same hospital. All of them had normal renal function, normal urinalysis results, and no personal or family history of nephropathy or other serious illness. 
Finally, 120 patients with IgAN, 45 patients with MN, 31 patients with MCD and 40 healthy volunteers were enrolled in this study. Among the IgAN patients, the first 6 patients with grade I-II according to Lee's grading system who met the inclusion criteria and a sex ratio of 1 to 1 were selected as a screening cohort, as well as 6 patients with grade III and 6 patients with grade IV-V. In addition, 14 age- and gender-matched controls which included 4 patients with MN, 4 patients with MCD and 6 healthy volunteers were selected in the screening cohort.

The demographic and clinical data, such as age, gender, mean aortic pressure (MAP), serum creatinine (Scr) and 24-hour urinary protein excretion (UPE), of all included participants were recorded at the time of kidney biopsy. The estimated glomerular filtration rate (eGFR) was calculated using the Chronic Kidney Disease Epidemiology Collaboration (CKD - EPI) equations (Levey et al. 2009).

\section{Evaluation of renal pathological lesion}

Renal tissues obtained by biopsy were stained with haematoxylin and eosin (H \&E), periodic acid Schiff (PAS), periodic acid silver methenamine (PASM) and Masson's trichrome for light microscopy and with lgG, IgA, IgM, C3 and C1q for immunofluorescence. Two pathologists who were blinded to patients' data evaluated the renal biopsy slides separately.

For patients with IgAN, the severity of renal pathological lesions were evaluated according to Lee's grading system (Lee et al. 1982) and Oxford classification (Roberts et al. 2009). First, all cases of IgAN were categorized into grade I-V according to Lee's grading system. Then, four variables of Oxford classification were further evaluated; that is, mesangial proliferation (M1 - more than half the glomeruli have more than three cells in a mesangial area, M0 - not meet the criteria of M1), endocapillary hypercellularity (E1 - present, E0 - absent), segmental glomerulosclerosis (S1 - present, S0 - absent ), and tubular atrophy/ interstitial fibrosis (T0 - 0\%-25\%, T1 - 26\% - 50\%, T2 - > 50\%).

\section{Urine sample preparation}


A whole-stream early morning urine specimen was collected at the day of renal biopsy. The urine sample was centrifuged at $3,000 \mathrm{~g}$ for $30 \mathrm{~min}$ and at $13,000 \mathrm{~g}$ for $5 \mathrm{~min}$ at $4^{\circ} \mathrm{C}$; supernatant was discarded (Wang et

127 al. 2012; Wang \& Szeto 2013). Then the urinary cell pellet was lysed by RNA lysis buffer MZ (Tiangen

128 Biotech, Beijing, China, catalog number DP501) and stored at $-80^{\circ} \mathrm{C}$ until used.

129

130

131

132

133

134

135

136

137

138

139

140

141

142

143

144

145

146

147

\section{RNA extraction}

Total RNA was extracted using a miRcute miRNA Isolation Kit (Tiangen Biotech, Beijing, China, catalog number DP501) according to the manufacturer's protocol. The quantity ( $\mathrm{ng} / \mathrm{ml}$ ) and purity (ratio of absorbances of the RNA isolates at $260 \mathrm{~nm}$ and $280 \mathrm{~nm}$ [A260/280]) of the RNAs obtained was evaluated by NanoDrop 2000 spectrophotometer (Thermo Scientific, USA).

\section{MiRNA microarray analysis}

Affymetrix GeneChip miRNA 4.0 Array (Affymetrix, US, catalog number 902411), which covers all of the 2,578 mature human miRNAs available in miRBase version 20 (June 24, 2013, www.mirbase.org/) (Kozomara \& Griffiths-Jones 2011), was used to profile miRNA expressions. Briefly, 1 mg of RNA was polyA tailed and labelled with a FlashTag Biotin HSR RNA Labeling Kit (Affymetrix, US, catalog number 901911). The labelled RNA was hybridized at $48^{\circ} \mathrm{C}$ for $16 \mathrm{hr}$ on the miRNA arrays, which were washed and stained with Affymetrix Fluidics Station 450 (Affymetrix, US) and scanned with an Affymetrix GeneChip Scanner 3000 (Affymetrix, US) using the Command Console software (Affymetrix, US). The data were analyzed with miRNA QCTool (Affymetrix, US) using the Affymetrix default analysis settings and quantile as the normalization method. The microarray data have been deposited in Gene Expression Omnibus (GEO) at NCBI ( http://www.ncbi.nlm.nih.gov/geo/ ) with accession number GSE64306.

\section{RT-qPCR analysis}

RT-qPCR was carried out in compliance with the MIQE guidelines (Bustin et al. 2009) to verify the candidate miRNAs revealed by microarray. Briefly, reverse transcription was performed using $500 \mathrm{ng}$ of total 
148 RNA and a miRcute miRNA First-Strand cDNA Synthesis Kit (Tiangen Biotech, Beijing, China, catalog

149 number KR201) according to the manufacturer's protocol. RT-qPCR was performed on an Applied

150 Biosystems 7500 Fast Dx Real-Time PCR Instrument (Applied Biosystems, USA) using a miRcute miRNA

151 qPCR Detection Kit (Tiangen Biotech, Beijing, China, catalog number FP401), according to the

152 manufacturer's instructions. All of the primers were purchased from Tiangen Biotech Company (Beijing,

153 China). All PCR reactions were performed in triplicate, followed by melt curve analysis to verify their

154 specificity and identity. U6 was selected as the endogenous reference control (Mestdagh et al. 2009).

155 Relative miRNA expression levels were calculated using the $\Delta \Delta \mathrm{Ct}$ method as previously described (Livak \&

156 Schmittgen 2001).

157 MiRNA target prediction and function analysis

158 The candidate miRNAs were imported into the miRWalk algorithm (Dweep et al. 2011) (

159 http://www.umm.uni-heidelberg.de/apps/zmf/mirwalk/ ), and the prediction of their target genes was

160 performed using nine additional algorithms, including TargetScan, DIANAmT, miRanda, miRDB, RNAhybrid,

161 PICTAR4, PICTAR5, PITA and RNA22.

To explore the functional annotation and pathway enrichment of those predicted genes, the Gene

163

164

165

166

167

168

169

170

171

Ontology (GO) and KEGG (Kyoto Encyclopedia of Genes and Genomes) database analyses were conducted using a DAVID (the Database for Annotation, Visualization and Integrated Discovery) online analysis tool (Huang da et al. 2009) ( http://david.abcc.ncifcrf.gov/tools.jsp ), in which we focused on the GO biology process (BP) feature.

\section{Statistical analysis}

The microarray data were analyzed by the algorithm of SAM (significance analysis of microarrays, wwwstat.stanford.edu/ tibs/SAM/) (Olson 2006). The false discovery rate (FDR) was set to $<0.05$ and the minimum fold change $(\mathrm{FC})$ was set to $>2.0$ or $<0.5$. Hierarchical clustering was carried out using the $\mathrm{MeV}$ 4.9 software (Multi Experiment Viewer, http://www.tm4.org/mev.htm) to generate both miRNA and sample 
172 trees based on Pearson correlation. For the RT-qPCR data, statistical analysis was performed by SPSS

173 version 20.0 (SPSS, USA). Data were compared by Mann-Whitney $U$ test, Kruskal-Wallis test and

174 Spearman's rank order correlations as appropriate. In bioinformatics analysis, the Fisher's exact test and $x^{2}$

175 test were used to select the significant GO category or KEGG pathway, and the FDR was calculated to 176 correct the $P$ value. A $P$-value $<0.05$ was considered to be statistically significant.

Results

178

179

180

181

182

183

184

185

186

187

188

189

190

191

192

193

194

\section{Patients characteristics}

Demographic and clinical characteristics of the screening and validation cohorts at the time of renal biopsy are provided in Table 1 and Table 2 respectively. There were no significant differences in age and MAP among different groups in both cohorts.

In the screening cohort, the levels of UPE in IgAN grade I-II and III subgroups were lower than that in MCD group (all $P<0.01$ ) and higher than that in healthy control $(\mathrm{HC})$ group (all $P<0.01$ ); the levels of Scr in IgAN grade IV-V subgroup were higher than that in both $\mathrm{MN}$ and $\mathrm{HC}$ group (all $P<0.05$ ), whereas the levels of eGFR showed an opposite trend.

In the validation cohort, the levels of UPE in IgAN group were lower than that in MN and MCD group (all $P<0.01)$, but higher than that in $\mathrm{HC}$ group $(P<0.001)$; the levels of Scr were higher than that in all control groups (all $P<0.01$ ), whereas the levels of eGFR showed an opposite trend. The histopathological parameters are shown in Table 3.

\section{The yield of RNA from urinary sediments}

RNA yield from urinary sediments (ng of RNA isolated from per ml of urine) was determined and showed no significant differences among patients with $\operatorname{IgAN}, \mathrm{MN}, \mathrm{MCD}$ and healthy subjects. The overall median was $22.73 \mathrm{ng} / \mathrm{ml}$ (interquartile range $[I Q R]=10.75$ - 40.35). A260/280 was also recorded and showed no significant differences among different groups in the present study. The overall mean of A260/280 was 1.95 
(range $=1.82-2.07, \mathrm{SD}=0.06)$

\section{Differentially expressed miRNAs in the screening phase}

197

\section{Global miRNA profiling from microarray analysis}

To identify miRNAs differentially expressed in IgAN, miRNA microarrays were used to analyze their global expression profile of the four groups (IgAN, MN, MCD and HC). The 32 urine samples of the screening cohort were profiled on Affymetrix GeneChip miRNA 4.0 Arrays.

Among 2,578 mature human miRNAs represented on the microarray, $780(30.3 \%)$ miRNAs were identified as expressed in at least one urine sample. Unsupervised hierarchical clustering analysis demonstrated that miRNA profiling clearly differentiated IgAN samples from healthy controls; there were, however, one MN sample and two MCD samples mixed with IgAN, indicating that the differences between IgAN patients and disease controls were not as clear (Figure 1).

\section{Differentially expressed miRNAs in IgAN}

The microarray data were analyzed by SAM; the results identified various differentially expressed miRNAs that presented a $\mathrm{FC}>2$ or $<0.5$ and $P$ value $<0.05$ in urinary sediments of IgAN samples. Among them, there were 117 miRNAs differentially expressed between IgAN and HC (Table S1); 78 miRNAs differentially expressed between IgAN and MN (Table S2); and 11 miRNAs differentially expressed between IgAN and MCD (Table S3). There was not a single miRNA, however, that showed significantly different expression across all four groups.

\section{Differentially expressed miRNAs in IgAN subgroups}

In the screening phase, we grouped IgAN grade I-II as the early pathological change group; grade III as the mild pathological change group; and grade IV-V as the severe group according to Lee's grading system. Then we compared the different subgroups of IgAN (grade I-II, III and IV-V) with both healthy and 
217 disease controls. The differentially expressed miRNAs are shown in the form of volcano plot (Figure S1) and

218 Venn diagram (Figure 2). All these miRNAs presented a FC $>2$ or $<0.5$ and $\mathrm{P}$ value $<0.05$.

In the IgAN grade I-II subgroup, two miRNAs (hsa-miR-223-3p, hsa-miR-629-5p) were in high levels and two (hsa-miR-3613-3p, hsa-miR-4668-5p) were in low levels compared to all of the control groups

221 (Figure 2A). In the IgAN grade III subgroup, three miRNAs (hsa-miR-150-5p, hsa-miR-572 and hsa-miR$222371 b-5 p)$ were in high levels and three ( $h s a-m i R-3613-3 p$, hsa-miR-4668-5p and $h s a-m i R-6750-5 p$ ) were in 223 low levels compared to all control groups (Figure 2B). IgAN grade I-II and III subgroups shared dysregulation 224 of only two miRNAs (miR-3613-3p and miR-4668-5p). Interestingly, there were no overlaps in changes to 225 miRNAs for IgAN grade IV-V subgroup as shown in the Venn diagram (Figure 2C). It turned out that miRNAs 226 were in different levels only in specific IgAN subgroups.

\section{Validation of differentially expressed miRNAs using RT-qPCR}

228

229

230

231

232

233

234

To check the accuracy of the microarray-based miRNA quantification, the eight above-mentioned miRNAs (miR-223-3p, miR-629-5p, miR-150-5p, miR-572, miR-371b-5p, miR-3613-3p, miR-4668-5p and miR-6750-5p) were re-examined using RT-qPCR in the 32 samples of the screening cohort. Then we analyzed correlations between $\log _{2}$-transformed microarray signal and RT-qPCR Ct values.

As shown in Table 4, seven of the eight miRNAs (miR-223-3p, miR-629-5p, miR-150-5p, miR-572, miR371b-5p, miR-3613-3p and miR-4668-5p) demonstrated significant correlations $(|r|>0.7, P<0.01)$ between the two sets of measurements, indicating that the microarray data was relatively reliable.

\section{Analysis of candidate miRNAs in the validation phase}

\section{Validation of candidate miRNAs using RT-qRCR}

For confirmation purposes, the two miRNAs (miR-3613-3p and miR-4668-5p) that were differentially expressed in both IgAN grade I-II and III subgroups in the screening phase were selected as candidates and analyzed in the validation cohort, which included 102 patients with IgAN, 41 patients with MN, 27 patients 
with MCD and 34 healthy controls.

RT-qPCR results showed that the levels of $m i R-3613-3 p$ were significantly lower in IgAN as compared with that in $\mathrm{HC}, \mathrm{MN}$ and MCD, respectively $[0.27$ ( $0.19-0.38)$ vs 1.10 ( $0.9-1.26$ ), 0.58 ( $0.32-1.00$ ) and 0.61 ( $0.39-0.77$ ), respectively, all $P<0.01$ ] (Figure 3A); the levels of miR-4668-5p were significantly lower in IgAN than that in $\mathrm{HC}[0.54(0.38-0.60)$ vs $1.11(0.73-1.22), P=0.001]$; there were no significant differences for miR-4668-5p, however, between IgAN and the disease controls (Figure 3B).

\section{Correlations between candidate miRNAs and clinical parameters}

Correlations between urinary levels of candidate miRNAs and clinical parameters, such as UPE, Scr, eGFR and Lee's grades, were further analyzed within the IgAN group (Table 5). Urinary levels of miR-3613$3 p$ were positively correlated with both UPE $(r=0.50, P=0.034)$ and Lee's grades $(r=0.57, P=0.014)$, while negatively correlated with eGFR $(r=-0.48, P=0.043)$. Similarly, significant correlations were found between urinary levels of miR-4668-5p with eGFR $(r=-0.50, P=0.034)$ and Lee's grades $(r=0.57, P=$ 0.013).

In addition, relationships between expressions of candidate miRNAs and four components of Oxford classification are shown in Figure 4. For segmental glomerulosclerosis, patients scored as SO had significantly lower levels of urinary $m i R-3613-3 p$ and $m i R-4668-5 p$ than those scored as S1 [0.23 (0.12 $0.33)$ vs $0.56(0.29-2.50), 0.23(0.18-0.25)$ vs $0.53(0.35-1.90)$, respectively, all $P<0.05]$.

\section{Bioinformatics analysis of candidate miRNAs}

\section{Predicted target genes of candidate miRNAs}

Only 3 out of 10 algorithms of miRWalk (DIANAmT, miRDB and Targetscan) can query the predicted target genes of $m i R-3613-3 p$ and $m i R-4668-5 p$. For $m i R-3613-3 p$, there were 5138, 2292 and 1249 target genes predicted by DIANAmT, miRDB and Targetscan, respectively. For miR-4668-5p, there were 1766, 1169 and 552 target genes predicted by DIANAmT, miRDB and Targetscan, respectively. To reduce 
263 redundancy, only genes predicted by all three algorithms were selected for further analysis; that is, 593

264 predicted targets genes for $m i R-3613-3 p$ and 175 predicted targets genes for $m i R-4668-5 p$ (data not shown).

\section{Functions and pathway analysis of miRNAs targets}

266 To explore in-depth biological information, the GO and KEGG database analyses were conducted using

a DAVID online analysis tool. All of the significant GO Biological Processes for $m i R-3613-3 p$ and $m i R-4668$ -

$2685 p$ are listed in Table S4 and Table S5 respectively; the top 20 GO Biological Processes according to $P$ 269 values and all significant KEGG pathways for miR-3613-3p and miR-4668-5p are shown in Figure 5 and 270 Figure 6 respectively.

There were 254 and 49 significant GO terms for miR-3613-3p and miR-4668-5p respectively $(P<0.05)$.

272 The most significantly enriched GO terms for miR-3613-3p were the regulation of transcription (GO: 0045449, 273 GO: 0006350) and macromolecule metabolic process (GO: 0010604, GO: 0010557) as shown in Figure 5A; 274 it also had significant enrichment in B cell differentiation (GO: 0030183, $P=0.006$ ) and activation (GO: $2750042113, P=0.016$ ) as listed in Table S4. Similarly, the most significantly enriched GO terms for miR-4668$2765 p$ (as shown in Figure 6A) also included transcription (GO: 0006350, GO: 0045449) and macromolecule metabolic process (GO: 0051252, GO: 0010558), as well as nuclear transport (GO: 0006607, GO: 0051169).

In addition, as shown in Figure 6, there were 18 and 2 significant KEGG pathways for miR-3613-3p and miR-4668-5p respectively $(P<0.05)$; both included Wnt signaling pathway (hsa04310, $P=0.012$ and $P=$ 0.013 respectively).

\section{Discussion}

Genome-wide analyses of miRNA expressions in peripheral blood mononuclear cells (PBMCs) and kidney biopsy tissues have identified a number of miRNAs differentially expressed in patients with IgAN compared to healthy controls (Serino et al. 2012; Tan et al. 2013). In the present study, our microarray data also showed that there were a number of miRNAs that were up-regulated in urinary sediments of IgAN 
286

patients when compared with healthy subjects. However, the differentially expressed miRNAs identified in the three studies were not completely consistent with each other, which may be due to the use of different tissues and/or the possible effect of the different races of the subjects. It is therefore important to investigate miRNA profiles from different races in a larger study.

Wang et al. (Wang et al. 2011; Wang et al. 2012) quantified urinary levels of several miRNAs in 43 patients with IgAN and 13 healthy volunteers and found that the levels of $m i R-146 a, m i R-155$ and $m i R-93$ of IgAN were significantly higher than those of healthy controls, which are consistent with our microarray results. But these studies lacked disease controls to prove whether the differentially expressed miRNAs are diseasespecific for IgAN. In theory, any aberrant miRNA expression observed in IgAN patients could be either disease-specific or present in all patients with chronic kidney disease (Szeto \& Li 2014). Thus, in the present study, we recruited patients with MN or MCD as other glomerulopathy controls and explored the whole urinary miRNA profile in IgAN patients.

Analyses of our microarray data demonstrated that although the urinary miRNA profilings of $\lg A N$ samples were clearly differentiated from healthy controls, they were mixed to a certain degree with disease controls; that is, no miRNA was found to be disease-specific for IgAN at the screening phase of this study. Thus, we performed subgroup analyses according to Lee's grading system, the results showed that eight miRNAs were differentially expressed either in the IgAN grade I-II subgroup or in the IgAN grade III subgroup compared to all control groups, but no miRNA was specific in the IgAN grade IV-V subgroup. This result may be explained by considerable histologic variability of IgAN, which ranges from no detectable histologic lesion to diffuse proliferative glomerulonephritis, and these cumulative changes may eventually results in glomerulosclerosis and tubulointerstitial fibrosis that are common in patients with chronic kidney disease (Haas \& Reich 2012; Loeffler \& Wolf 2014).

When studying the validation cohort, we verified that miR-3613-3p was in significantly low levels in urinary sediments of IgAN patients compared to healthy controls and MN or MCD patients, indicating it may have potential diagnostic value for IgAN. But it is necessary to note that the pathological grades of IgAN 
311 patients enrolled in this study were predominantly grade II ( $16.7 \%$ ), III ( $51.0 \%$ ), and IV ( $23.5 \%$ ) according

312 to Lee's grading system, while grades I ( $3.9 \%)$ and V ( $4.9 \%)$ were rare. This phenomenon is also consistent

313 with previous studies (Bartosik et al. 2001; Lee et al. 2005). In the present study, we also observed that the

314 expression levels of miR-3613-3p and miR-4668-5p were significantly correlated with eGFR, Lee's grades

315 and glomerulosclerosis, indicating that there may be a possible correlation between the levels of the two 316 miRNAs and the severity of IgAN.

New evidences are emerging that $\lg \mathrm{AN}$ is an immune-mediated disease with galactose-deficient $\lg \mathrm{A} 1$, which can elicit an autoantibody response and formation of immune complexes that are deposited in the mesangium (Kiryluk \& Novak 2014; Pillai et al. 2014). Although miR-3613-3p and miR-4668-5p were reported with unknown biological functions, bioinformatics analysis may shed light on the roles of these miRNAs by predicting miRNA-regulated genes. According to the GO analysis, the predicted functions of both miR-3613$3 p$ and $m i R-4668-5 p$ were significantly enriched in regulation of transcription and macromolecule metabolic process, which is consistent with the study of Tan et al (Tan et al. 2013). Moreover, the gene functions predicted by miR-3613-3p was also significantly enriched in B cell differentiation and activation, which may be related to the mechanism of IgAN.

According to the KEGG analysis, the predicted pathways of both miR-3613-3p and miR-4668-5p were significantly enriched in Wnt signaling pathway. It was reported previously that Wnt proteins are mitogenic for pro-B cells (Reya et al. 2000). Moreover, a recent study has shown that the Wnt pathway was hyperactivated in PBMCs of IgAN patients, and the proliferation rate of PBMCs isolated from IgAN patients was significantly enhanced (Cox et al. 2010). Therefore, we make a hypothesis that the decreased expression of miR-3613-3p might regulate the activation of $\mathrm{B}$ cells through Wnt pathway, and participate in the pathogenesis of IgAN, providing a direction for further functional studies. determining their cellular source. MiRNAs in urinary sediments presumably reflect cellular miRNA content, 335 including podocytes, inflammatory cells, deciduous renal tubular epithelial cells and cells from urinary tract, 
but not including exosomes because of the relatively low $g$ values during centrifugation. Future studies would needs further investigation. patients, and the microarray data provided a valuable repertoire to discover non-invasive biomarkers for IgAN. Further studies with larger numbers of patients and controls, especially in different races, are urgently needed to validate the expression profiles. Urinary expression of miR-3613-3p was down-regulated in patients with IgAN, and the levels of both miR-3613-3p and miR-4668-5p were correlated with disease severity. Further studies are needed to explore the roles of miR-3613-3p and miR-4668-5p in the pathogenesis and progression of IgA nephropathy.

\section{References}

Bartosik LP, Lajoie G, Sugar L, and Cattran DC. 2001. Predicting progression in IgA nephropathy. Am J Kidney Dis 38:728-735. Bustin SA, Benes V, Garson JA, Hellemans J, Huggett J, Kubista M, Mueller R, Nolan T, Pfaffl MW, Shipley GL et al. . 2009. The MIQE guidelines: minimum information for publication of quantitative real-time PCR experiments. Clin Chem 55:611-622.

Chandrasekaran K, Karolina DS, Sepramaniam S, Armugam A, Wintour EM, Bertram JF, and Jeyaseelan K. 2012. Role of microRNAs in kidney homeostasis and disease. Kidney Int 81:617-627.

Chen X, Ba Y, Ma L, Cai X, Yin Y, Wang K, Guo J, Zhang Y, Chen J, Guo X et al. . 2008. Characterization of microRNAs in serum: a novel class of biomarkers for diagnosis of cancer and other diseases. Cell Res 18:997-1006.

Cox SN, Sallustio F, Serino G, Pontrelli P, Verrienti R, Pesce F, Torres DD, Ancona N, Stifanelli P, Zaza G et al. . 2010. Altered modulation of WNT-beta-catenin and PI3K/Akt pathways in IgA nephropathy. Kidney Int 78:396-407.

Dweep H, Sticht C, Pandey P, and Gretz N. 2011. miRWalk--database: prediction of possible miRNA binding sites by "walking" the genes of three genomes. J Biomed Inform 44:839-847.

Esteller M. 2011. Non-coding RNAs in human disease. Nat Rev Genet 12:861-874.

Haas M, and Reich HN. 2012. Morphologic markers of progressive immunoglobulin A nephropathy. Adv Chronic Kidney Dis 19:107-113.

Huang da W, Sherman BT, and Lempicki RA. 2009. Systematic and integrative analysis of large gene lists using DAVID bioinformatics resources. Nat Protoc 4:44-57.

Kiryluk K, and Novak J. 2014. The genetics and immunobiology of IgA nephropathy. J Clin Invest 124:2325-2332.

Kozomara A, and Griffiths-Jones S. 2011. miRBase: integrating microRNA annotation and deep-sequencing data. Nucleic Acids Res 39:D152-157. 
Lee HS, Lee MS, Lee SM, Lee SY, Lee ES, Lee EY, Park SY, Han JS, Kim S, and Lee JS. 2005. Histological grading of IgA nephropathy predicting renal outcome: revisiting H. S. Lee's glomerular grading system. Nephrol Dial Transplant 20:342-348.

Lee SM, Rao VM, Franklin WA, Schiffer MS, Aronson AJ, Spargo BH, and Katz Al. 1982. IgA nephropathy: morphologic predictors of progressive renal disease. Hum Pathol 13:314-322.

Levey AS, Stevens LA, Schmid CH, Zhang YL, Castro AF, 3rd, Feldman HI, Kusek JW, Eggers P, Van Lente F, Greene T et al. . 2009. A new equation to estimate glomerular filtration rate. Ann Intern Med 150:604-612.

Livak KJ, and Schmittgen TD. 2001. Analysis of relative gene expression data using real-time quantitative PCR and the 2(Delta Delta C(T)) Method. Methods 25:402-408.

Loeffler I, and Wolf G. 2014. Transforming growth factor-beta and the progression of renal disease. Nephrol Dial Transplant 29 Suppl 1:i37-i45.

Mestdagh P, Van Vlierberghe P, De Weer A, Muth D, Westermann F, Speleman F, and Vandesompele J. 2009. A novel and universal method for microRNA RT-qPCR data normalization. Genome Biol 10:R64.

Olson NE. 2006. The microarray data analysis process: from raw data to biological significance. NeuroRx 3:373-383.

Pillai U, Balabhadraputani K, and Bhat Z. 2014. Immunoglobulin A nephropathy: a review of current literature on emerging pathophysiology. Am J Med Sci 347:249-253.

Reya T, O'Riordan M, Okamura R, Devaney E, Willert K, Nusse R, and Grosschedl R. 2000. Wnt signaling regulates B lymphocyte proliferation through a LEF-1 dependent mechanism. Immunity 13:15-24.

Roberts IS, Cook HT, Troyanov S, Alpers CE, Amore A, Barratt J, Berthoux F, Bonsib S, Bruijn JA, Cattran DC et al. . 2009. The Oxford classification of IgA nephropathy: pathology definitions, correlations, and reproducibility. Kidney Int 76:546556.

Schena FP. 1990. A retrospective analysis of the natural history of primary IgA nephropathy worldwide. Am J Med 89:209215.

Serino G, Sallustio F, Cox SN, Pesce F, and Schena FP. 2012. Abnormal miR-148b expression promotes aberrant glycosylation of IgA1 in IgA nephropathy. J Am Soc Nephrol 23:814-824.

Szeto CC, and Li PK. 2014. MicroRNAs in IgA nephropathy. Nat Rev Nephrol 10:249-256.

Tan K, Chen J, Li W, Chen Y, Sui W, Zhang Y, and Dai Y. 2013. Genome-wide analysis of microRNAs expression profiling in patients with primary IgA nephropathy. Genome 56:161-169.

Wang G, Kwan BC, Lai FM, Chow KM, Kam-Tao Li P, and Szeto CC. 2010. Expression of microRNAs in the urinary sediment of patients with IgA nephropathy. Dis Markers 28:79-86.

Wang G, Kwan BC, Lai FM, Chow KM, Li PK, and Szeto CC. 2011. Elevated levels of miR-146a and miR-155 in kidney biopsy and urine from patients with IgA nephropathy. Dis Markers 30:171-179.

Wang G, Kwan BC, Lai FM, Chow KM, Li PK, and Szeto CC. 2012. Urinary miR-21, miR-29, and miR-93: novel biomarkers of fibrosis. Am J Nephrol 36:412-418.

Wang G, and Szeto CC. 2013. Methods of microRNA quantification in urinary sediment. Methods Mol Biol 1024:211-220.

Wyatt RJ, and Julian BA. 2013. IgA nephropathy. N Engl J Med 368:2402-2414. 


\section{1}

Global urinary miRNA profiling in patients with IgAN and controls.

Unsupervised hierarchical clustering using $\log _{2}$-transformed microarray signal values of the 780 expressed miRNAs is shown as a heat-map. Columns represent urine samples and rows miRNAs. Urine samples were from 18 patients with IgAN (red), 6 healthy subjiects (orange), 4 patients with MN (dark blue) and 4 patients with MCD (light blue). Heat-map colours represent relative miRNA expression as indicated in the colour scale: yellow represents high expression; blue represents low expression; black respresents median expression level equal to 1 . HC, healthy control; IgAN, IgA nephropathy; I-II, III and IV-V, grade I-II, III and IV-V according to Lee's grading system, respectivley; MN, membranous nephropathy; MCD, minimal change disease. 


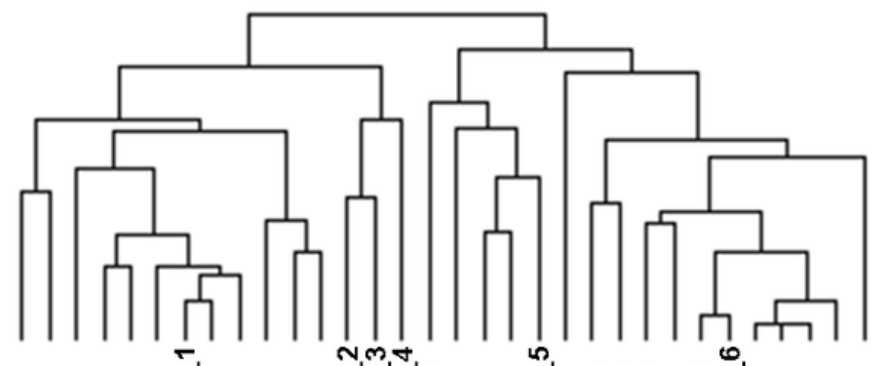

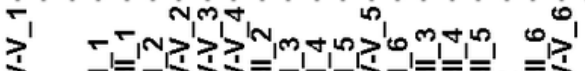

2.00

$\geq_{1}-m_{1}=$ -Nm, no

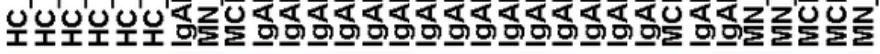

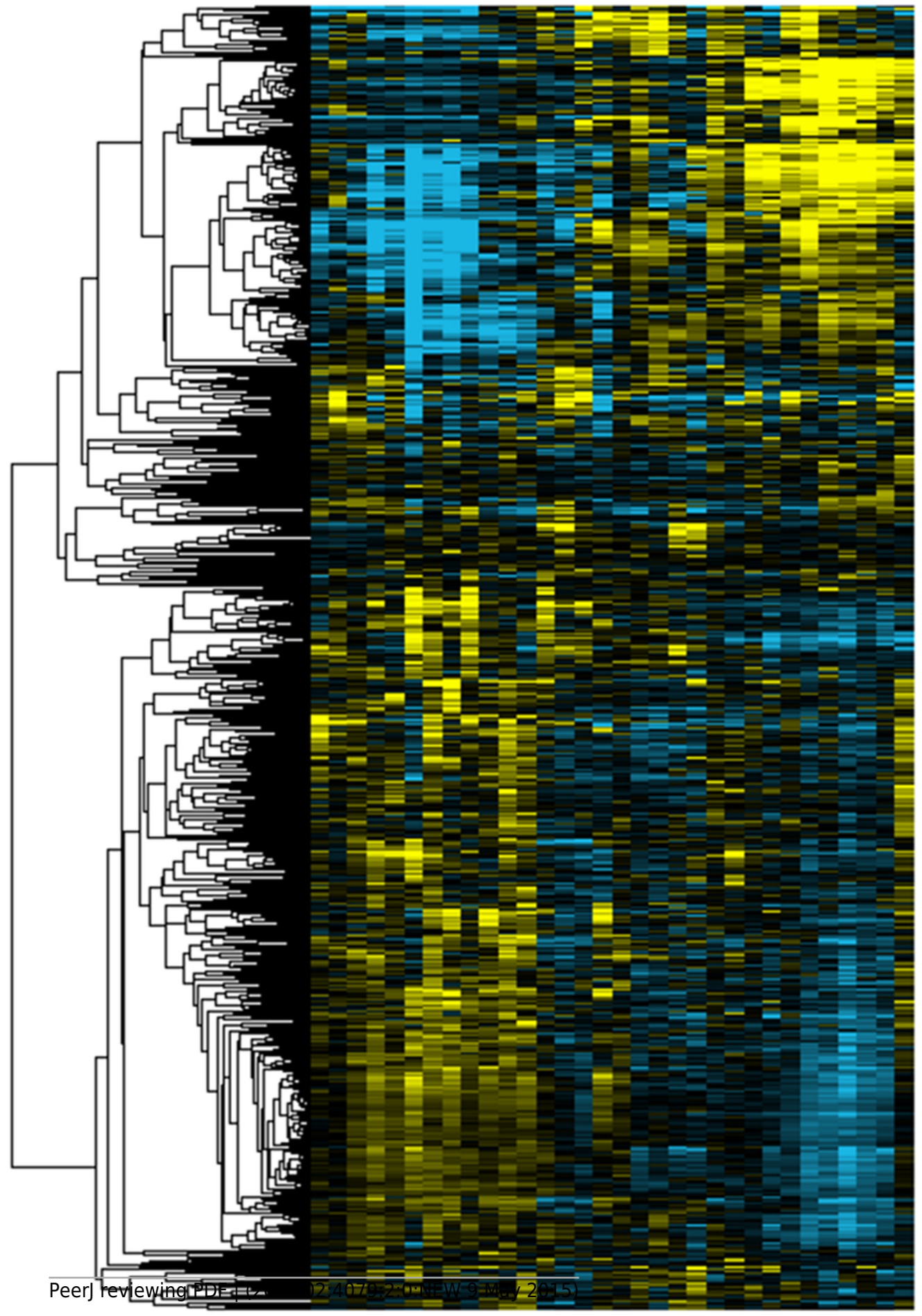




\section{2}

Overlapping relationship of the differentially expressed miRNAs in IgAN subgroups.

Urinary levels of miRNAs in the subgroups of IgAN (IgAN grade I-II, III and IV-V subgroup according to Lee's grading system) were compared with each control group (HC, MN and $M C D$ ) in the screening phase. All differentially expressed miRNAs presented a fold change $>$ 2 and $P$ value $<0.05$. The miRNAs in the centre of Venn diagram were (A) miR-223-3p, miR629-5p, miR-3613-3p and miR-4668-5p; (B) miR-150-5p, miR-572, miR-371b-5p, miR-3613$3 p$, miR-4668-5p and miR-6750-5p. IgAN, IgA nephropathy; HC, healthy control; MN, membranous nephropathy; MCD, minimal change disease.

A

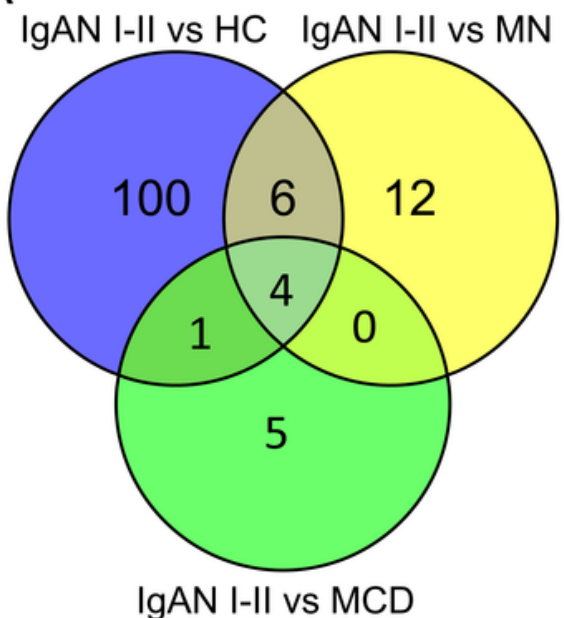

$B$ IgAN III vs HC IgAN III vs MN

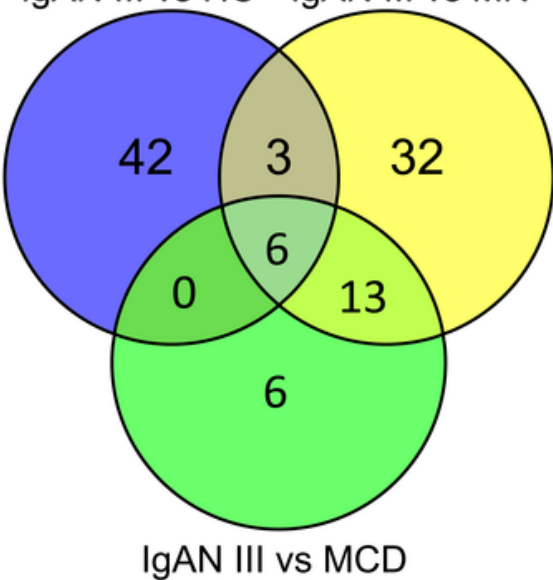

C IgAN IV-V vs HC IgAN IV-V vs MN

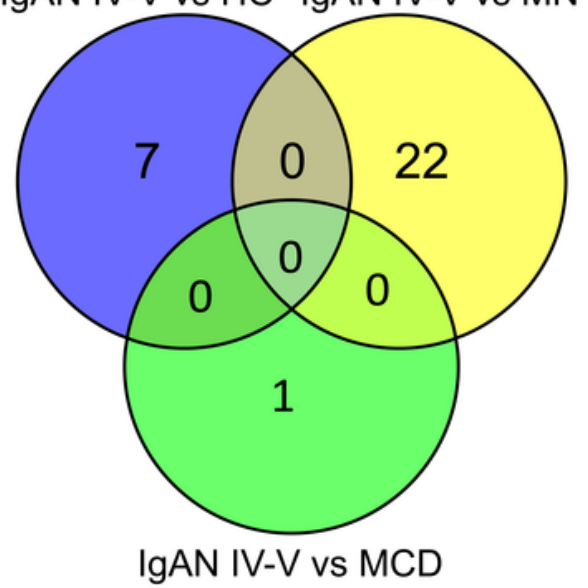




\section{3}

Comparison of candidate miRNAs levels between IgAN and each control group.

Urinary levels of candidate miRNAs were analyzed by RT-qPCR in the validation cohort, which including 102 patients with IgAN, 41 patients with MN, 27 patients with MCD and 34 healthy controls. The whisker-box plots depict the relative expression level of $m i R-3613-3 p$ and $m i R$ 4668-5p. The boxes indicate median and 25th and 75th percentiles; whisker caps indicate 5th and 95th percentiles. Statistically significant differences were determined by MannWhitney $\mathrm{U}$ test. Levels are represented as ratio to the median of healthy controls. NS, not significant ( $P>0.05$ ); IgAN, IgA nephropathy; $\mathrm{HC}$, healthy control; $\mathrm{MN}$, membranous nephropathy; $M C D$, minimal change disease. (A) Urinary miR-3613-3p levels was significantly lower in IgAN as compared with that in $\mathrm{HC}, \mathrm{MN}$ and $\mathrm{MCD}$, respectively $[0.27(0.19-0.38), \mathrm{n}$ $=102$ vs $1.10(0.9-1.26), \mathrm{n}=34 ; 0.58(0.32-1.00), \mathrm{n}=41$ and $0.61(0.39-0.77), \mathrm{n}=$ 27; respectively, all $P<0.01]$. (B) Urinary miR-4668-5p levels were significantly lower in IgAN than that in $\mathrm{HC}[0.54(0.38-0.60), \mathrm{n}=102$ vs $1.11(0.73-1.22), \mathrm{n}=34 ; P=0.001]$. Urinary miR-4668-5p levels there were were not different between IgAN and MN and MCD [0.54 ( $0.38-0.60), n=102$ vs $0.56(0.41-0.88), n=41$ and $0.65(0.49-0.87), n=27$; repectively]. 


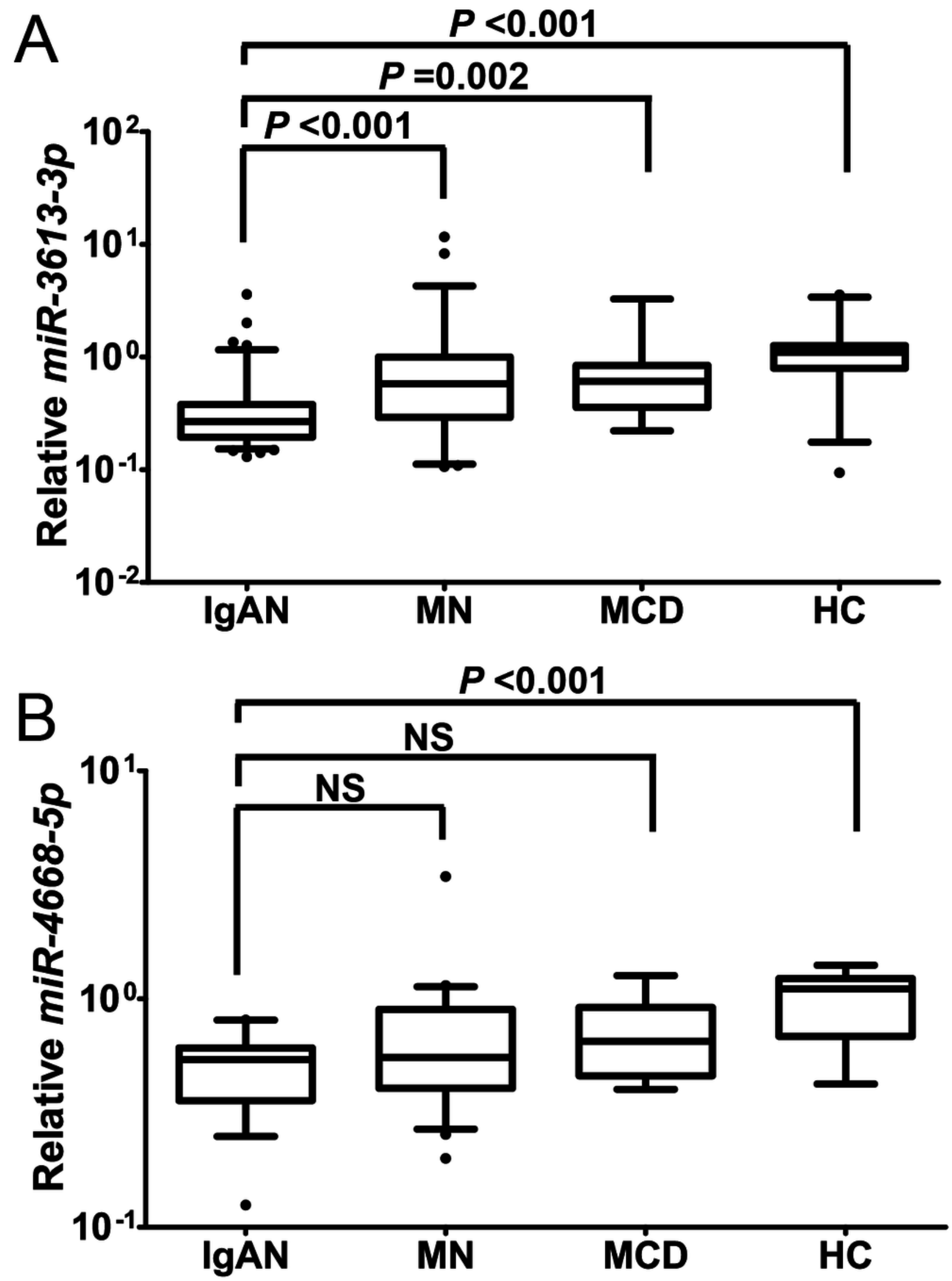




\section{4}

Relationships between candidate miRNAs levels and pathological parameters in patients with IgAN according to Oxford classification.

The whisker-box plots with medians and inter-quartile ranges depict the relative expression level of miR-3613-3p and miR-4668-5p. Data were analyzed by Mann-Whitney U test. IgAN, IgA nephropathy; HC, healthy control; MN, membranous nephropathy; $M C D$, minimal change disease. (A) Urinary miR-3613-3p levels of patients with S1 were higher than patients with S0 [0.56 ( $0.29-2.5), n=69$ vs $0.23(0.12-0.33), n=33 ; P=0.019]$. Urinary miR-3613-3p levels of patients with $\mathrm{M} 1 / \mathrm{MO}, \mathrm{E} 1 / \mathrm{E} 0$ and $\mathrm{T} 2 / \mathrm{T} 1 / \mathrm{TO}$ were not different between each other [0.28 ( $0.21-1.52), n=43$ vs $0.51(0.31-1.3), n=59 ; 0.55(0.26-1.61), n=13$ vs 0.49 ( $0.26-1.22), n=89 ; 2.58(0.81-9.18), n=9$ vs $0.72(0.5-1.3)$ vs $0.33(0.23-0.51), n=$ 56]. (B) Urinary miR-4668-5p levels of patients with $\mathrm{S} 1$ were higher than patients with $\mathrm{SO}$ [0.53 ( $0.35-1.9), \mathrm{n}=69$ vs $0.23(0.18-0.25), \mathrm{n}=33 ; \mathrm{P}=0.019$ ]. Urinary miR-4668-5p levels of patients with M1/M0, E1/E0 and T2/T1/TO were not different between each other [0.33 ( $0.26-1.55), n=43$ vs $0.41(0.3-0.94), n=59 ; 0.43(0.32-1.55), n=13$ vs 0.4( $0.24-0.94), n=89 ; 2.18(0.73-3.13), n=9$ vs $0.43(0.36-0.89)$ vs $0.35(0.23-0.41)$, $\mathrm{n}=56]$. 

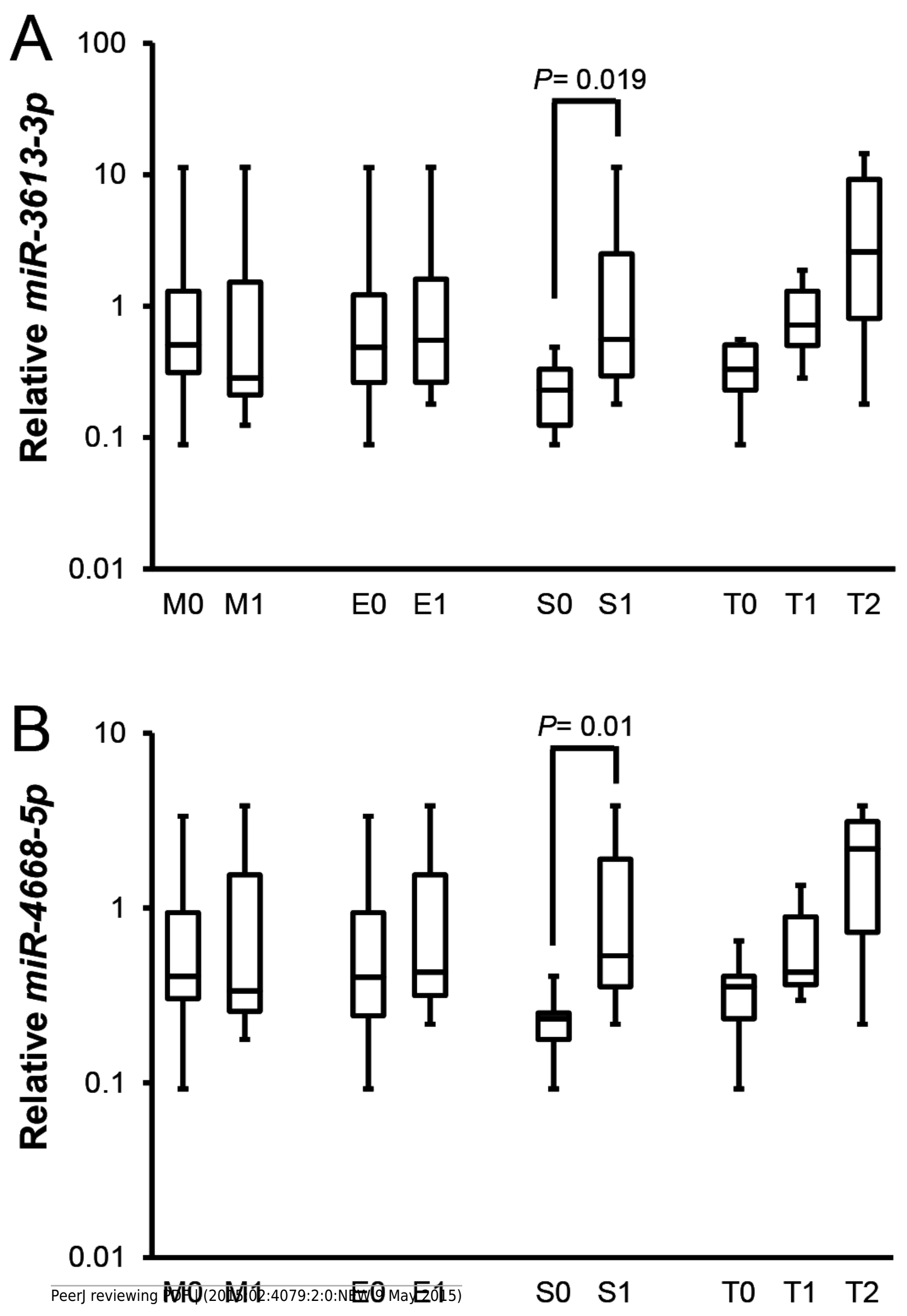


\section{5}

GO biology process and KEGG pathway enrichment analysis for predicted miRNA targets of $\operatorname{miR}-3613-3 p$.

GO biology process and KEGG pathway enrichments were performed by mapping the predicted target genes using DAVID online analysis tool. $P<0.05$ was used as a threshold to select significant GO terms and KEGG pathways. $\lg (P$ value $)$ is the negative $\log 10$ of the $P$ value. The the top $20 \mathrm{GO}$ biology processes accordign to $-\lg (P$ value) and a total of $18 \mathrm{KEGG}$ pathways for miR-3613-3p were annotated. GO, Gene Ontology; BP, biology process; KEGG, Kyoto Encyclopedia of Genes and Genomes. 


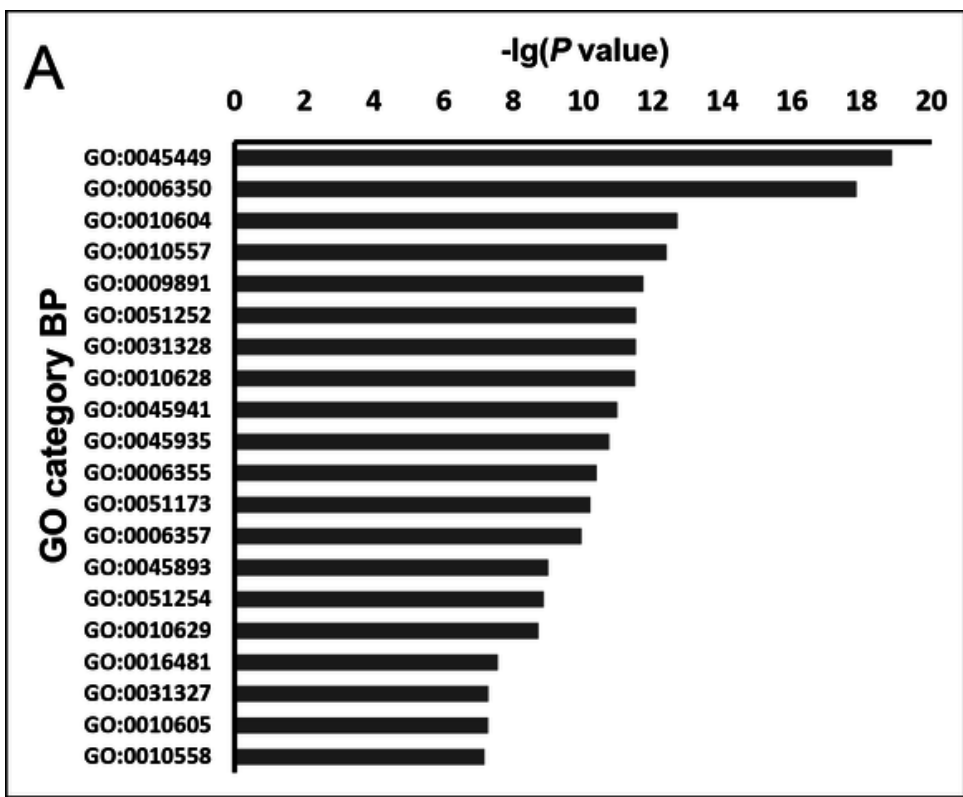

GO:0045449 regulation of transcription

GO:0006350 transcription

GO:0010604 positive regulation of macromolecule metabolic process GO:0010557 positive regulation of macromolecule biosynthetic process GO:0009891 positive regulation of biosynthetic process GO:0051252 regulation of RNA metabolic process GO:0031328 positive regulation of cellular biosynthetic process GO:0010628 positive regulation of gene expression GO:0045941 positive regulation of transcription

GO:0045935 positive regulation of nucleobase, nucleoside, nucleotide and nucleic acid metabolic process

GO:0006355 regulation of transcription, DNA-dependent

GO:0051173 positive regulation of nitrogen compound metabolic process GO:0006357 regulation of transcription from RNA polymerase II promoter GO:0045893 positive regulation of transcription, DNA-dependent GO:0051254 positive regulation of RNA metabolic process GO:0010629 negative regulation of gene expression GO:0016481 negative regulation of transcription GO:0031327 negative regulation of cellular biosynthetic process GO:0010605 negative regulation of macromolecule metabolic process GO:0010558 negative regulation of macromolecule biosynthetic process

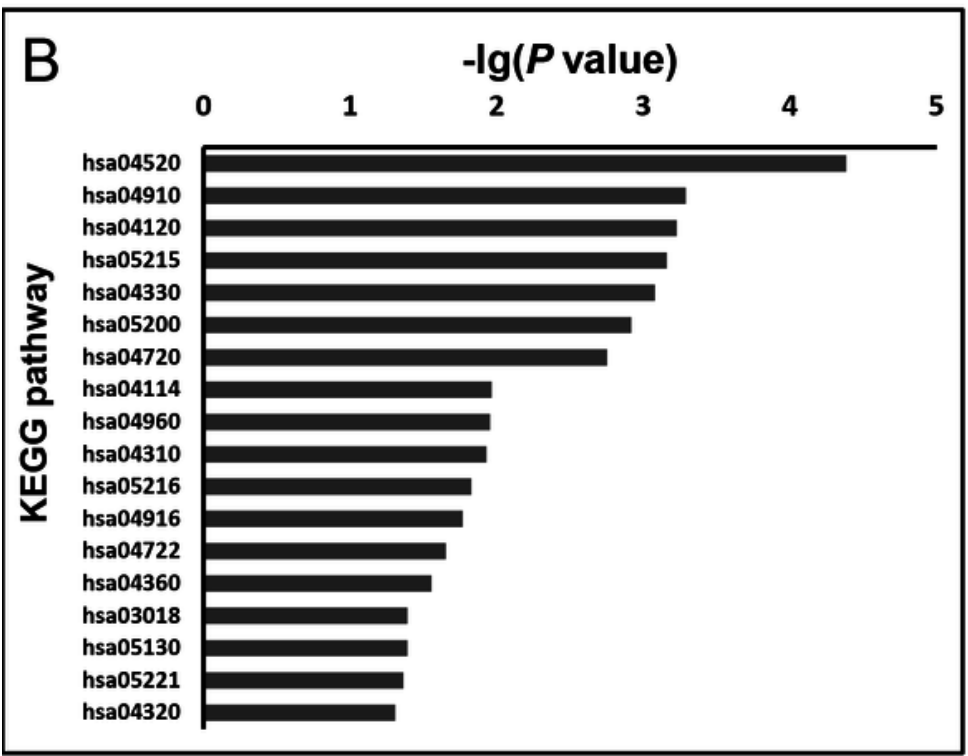

hsa04520 Adherens junction

hsa04910 Insulin signaling pathway

hsa04120 Ubiquitin mediated proteolysis

hsa05215 Prostate cancer

hsa04330 Notch signaling pathway

hsa05200 Pathways in cancer

hsa04720 Long-term potentiation

hsa04114 Oocyte meiosis

hsa04960 Aldosterone-regulated sodium reabsorption

hsa04310 Wnt signaling pathway

hsa05216 Thyroid cancer

hsa04916 Melanogenesis

hsa04722 Neurotrophin signaling pathway

hsa04360 Axon guidance

hsa03018 RNA degradation

hsa05130 Pathogenic Escherichia coli infection

hsa05221 Acute myeloid leukemia

hsa04320 Dorso-ventral axis formation 
GO biology process and KEGG pathway enrichment analysis for predicted miRNA targets of miR-4668-5p.

GO biology process and KEGG pathway enrichments were performed by mapping the predicted target genes using DAVID online analysis tool. $P<0.05$ was used as a threshold to select significant GO terms and KEGG pathways. $-\lg (P$ value $)$ is the negative $\log 10$ of the $P$ value. The the top $20 \mathrm{GO}$ biology processes accordign to $-\lg (P$ value $)$ and a total of 2 KEGG pathways for miR-4668-5p were annotated. GO, Gene Ontology; BP, biology process; KEGG, Kyoto Encyclopedia of Genes and Genomes.
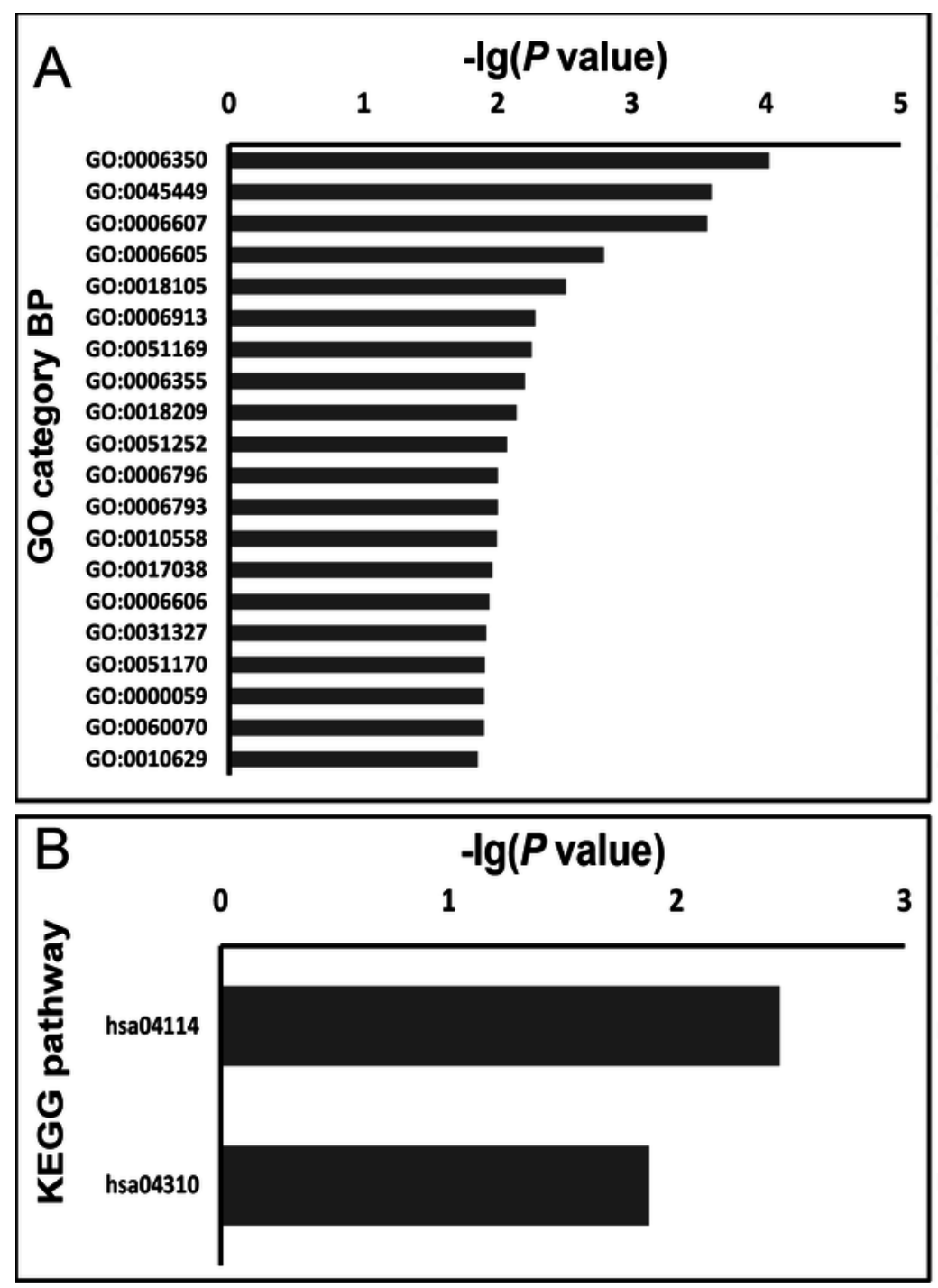

GO:0006350 transcription GO:0045449 regulation of transcription GO:0006607 NLS-bearing substrate import into nucleus GO:0006605 protein targeting GO:0018105 peptidyl-serine phosphorylation GO:0006913 nucleocytoplasmic transport GO:0051169 nuclear transport GO:0006355 regulation of transcription, DNA-dependent GO:0018209 peptidyl-serine modification GO:0051252 regulation of RNA metabolic process GO:0006796 phosphate metabolic process GO:0006793 phosphorus metabolic process GO:0010558 negative regulation of macromolecule biosynthetic process

GO:0017038 protein import

GO:0006606 protein import into nucleus

GO:0031327 negative regulation of cellular biosynthetic process GO:0051170 nuclear import GO:0000059 protein import into nucleus, docking GO:0060070 Wnt receptor signaling pathway through beta-catenin GO:0010629 negative regulation of gene expression 


\section{Table $\mathbf{1}_{\text {(on next page) }}$}

Demographic and clinical data of subjects in the screening cohort.

Continuous data are presented as mean \pm SEM; categorical data are presented as counts. IgAN, IgA nephropathy; MN, membranous nephropathy; MCD, minimal change disease; HC, healthy control; MAP, mean aortic pressure; UPE, 24-hour urinary protein excretion; Scr, serum creatinine; eGFR; estimated glomerular filtration rate. 
Table 1. Demographic and clinical data of subjects in the screening cohort.

\begin{tabular}{|c|c|c|c|c|c|c|}
\hline & IgAN I-II & IgAN III & $\lg A N$ IV-V & $\mathrm{MN}$ & MCD & $\mathrm{HC}$ \\
\hline Cases & 6 & 6 & 6 & 4 & 4 & 6 \\
\hline Gender (M/F) & $3 / 3$ & $3 / 3$ & $3 / 3$ & $2 / 2$ & $2 / 2$ & $3 / 3$ \\
\hline Age (years) & $27 \pm 5.3$ & $32.3 \pm 9.3$ & $36.3 \pm 10.5$ & $25.8 \pm 3.1$ & $33.3 \pm 8.5$ & $31.2 \pm 8.4$ \\
\hline MAP (mmHg) & $88.78 \pm 5.01$ & $90.89 \pm 2.93$ & $101 \pm 9.3$ & $91.42 \pm 10.85$ & $91.92 \pm 14.71$ & $87.21 \pm 4.87$ \\
\hline UPE (g/day) & $1.21 \pm 1.78$ & $0.82 \pm 0.75$ & $1.73 \pm 0.52$ & $4.17 \pm 1.66$ & $8.15 \pm 2.22$ & $0.008 \pm 0.003$ \\
\hline $\operatorname{Scr}(\mu \mathrm{mol} / \mathrm{L})$ & $78.7 \pm 20.22$ & $87.3 \pm 35.56$ & $126.23 \pm 23.12$ & $61.63 \pm 19.50$ & $82.58 \pm 25.63$ & $59.14 \pm 10.25$ \\
\hline $\begin{array}{l}\text { eGFR } \\
\left(\mathrm{ml} / \mathrm{min} / 1.73 \mathrm{~m}^{2}\right)\end{array}$ & $102.93 \pm 15.89$ & $94.64 \pm 23.97$ & $57.57 \pm 17.48$ & $129.29 \pm 17.54$ & $97.48 \pm 28.47$ & $135.46 \pm 12.87$ \\
\hline \multicolumn{7}{|c|}{ Oxford classification } \\
\hline M0/M1 & $6 / 0$ & $2 / 4$ & $3 / 3$ & - & - & - \\
\hline E0/E1 & $6 / 0$ & $5 / 1$ & $6 / 0$ & - & - & - \\
\hline So/S1 & $4 / 2$ & $1 / 5$ & $0 / 6$ & - & - & - \\
\hline $\mathrm{T} 0 / \mathrm{T} 1 / \mathrm{T} 2$ & $6 / 0 / 0$ & $3 / 2 / 1$ & $0 / 1 / 5$ & - & - & - \\
\hline
\end{tabular}

Continuous data are presented as mean \pm SEM; categorical data are presented as counts. IgAN, IgA nephropathy; MN, membranous nephropathy; MCD, minimal change disease; HC, healthy control; MAP, mean aortic pressure; UPE, 24hour urinary protein excretion; Scr, serum creatinine; eGFR; estimated glomerular filtration rate. 
Table 2 (on next page)

Demographic and clinical data of subjects in the validation cohort.

Continuous data are presented as mean \pm SEM; categorical data are presented as counts and/or proportions. IgAN, IgA nephropathy; MN, membranous nephropathy; MCD, minimal change disease; HC, healthy control; MAP, mean aortic pressure; UPE, 24-hour urinary protein excretion; Scr, serum creatinine; eGFR; estimated glomerular filtration rate. 
Table 2. Demographic and clinical data of subjects in the validation cohort.

\begin{tabular}{|c|c|c|c|c|c|c|c|}
\hline & IgAN & $\mathrm{MN}$ & MCD & $\mathrm{HC}$ & $\begin{array}{c}\text { IgAN } \\
\text { vs } \\
\text { MN }\end{array}$ & $\begin{array}{c}P \text { value } \\
\text { IgAN } \\
\text { vs } \\
\text { MCD }\end{array}$ & $\begin{array}{c}\text { IgAN } \\
\text { vs } \\
\text { HC }\end{array}$ \\
\hline Cases & 102 & 41 & 27 & 34 & - & - & - \\
\hline Gender (M/F) & $57(55.77 \%)$ & $24(58.54 \%)$ & $69(22.22 \%)$ & $17(50 \%)$ & 0.857 & 0.008 & 0.489 \\
\hline Age (years) & $35.87 \pm 11.82$ & $35.8 \pm 9.3$ & $31.6 \pm 10.9$ & $33.1 \pm 7.9$ & 0.935 & 0.963 & 0.659 \\
\hline $\operatorname{MAP}(\mathrm{mmHg})$ & $95.53 \pm 12.16$ & $94.17 \pm 9.72$ & $88.69 \pm 10.89$ & $86.59 \pm 7.35$ & 0.763 & 0.149 & 0.061 \\
\hline UPE (g/day) & $1.57 \pm 1.28$ & $4.61 \pm 2.61$ & $7.01 \pm 2.86$ & $0.015 \pm 0.013$ & $<0.001$ & $<0.001$ & $<0.001$ \\
\hline $\operatorname{Scr}(\mu \mathrm{mol} / \mathrm{L})$ & $105.89 \pm 51.68$ & $69.47 \pm 17.05$ & $78.08 \pm 22.12$ & $61.0 \pm 11.89$ & $<0.001$ & 0.003 & $<0.001$ \\
\hline $\begin{array}{l}\text { eGFR } \\
\left(\mathrm{ml} / \mathrm{min} / 1.73 \mathrm{~m}^{2}\right)\end{array}$ & $80.76 \pm 29.65$ & $119.62 \pm 18.77$ & $100.87 \pm 24.56$ & $126.54 \pm 10.85$ & $<0.001$ & 0.038 & $<0.001$ \\
\hline
\end{tabular}


Table 3(on next page)

Histopathological characteristics of patients with IgAN.

IgAN, IgA nephropathy. According to the Oxford classification, four variables were evaluated;

that is, mesangial proliferation (M), segmental glomerulosclerosis (S), endocapillary

hypercellularity $(\mathrm{E})$ and tubular atrophy/interstitial fibrosis $(\mathrm{T})$. 
Table 3. Histopathological characteristics of patients with IgAN.

\begin{tabular}{lcccccc}
\hline \multicolumn{1}{c}{ Oxford } & \multicolumn{5}{c}{ Lee's grade } & \\
\cline { 2 - 6 } \multicolumn{1}{c}{ classification } & $\mathrm{I}$ & $\mathrm{II}$ & $\mathrm{II}$ & $\mathrm{IV}$ & $\mathrm{V}$ & Total \\
\hline M0/M1 & $4 / 0$ & $17 / 0$ & $31 / 21$ & $6 / 18$ & $1 / 4$ & $59 / 43$ \\
E0/E1 & $4 / 0$ & $15 / 2$ & $43 / 9$ & $22 / 2$ & $5 / 0$ & $89 / 13$ \\
S0/S1 & $4 / 0$ & $14 / 3$ & $12 / 40$ & $3 / 21$ & $0 / 5$ & $33 / 69$ \\
T0/T1/T2 & $4 / 0 / 0$ & $14 / 3 / 0$ & $38 / 14 / 0$ & $0 / 20 / 4$ & $0 / 0 / 5$ & $56 / 37 / 9$ \\
Total & 4 & 17 & 52 & 24 & 5 & 102 \\
\hline
\end{tabular}

IgAN, IgA nephropathy. According to the Oxford classification, four variables were evaluated; that is, mesangial proliferation (M), segmental glomerulosclerosis (S), endocapillary hypercellularity (E) and tubular atrophy/interstitial fibrosis (T). 
Table 4 (on next page)

Correlations between miRNA quantification by microarray and RT-qPCR.

RT-qPCR, real-time quantitative polymerase chain reaction; $r$, Spearman correlation coefficient; $95 \% \mathrm{Cl}, 95 \%$ confidence interval. 
Table 4. Correlations between miRNA quantification by microarray and RT-qPCR.

\begin{tabular}{lccc}
\hline \multicolumn{1}{c}{ miRNA } & $\mathrm{r}$ & $95 \% \mathrm{Cl}$ & $P$ value \\
\hline miR-223-3p & -0.86 & $-0.93 \sim-0.72$ & $<0.001$ \\
miR-629-5p & -0.74 & $-0.87 \sim-0.52$ & $<0.001$ \\
miR-150-5p & -0.93 & $-0.97 \sim-0.8$ & $<0.001$ \\
miR-572 & -0.82 & $-0.91 \sim-0.68$ & $<0.001$ \\
miR-371b-5p & -0.77 & $-0.88 \sim-0.58$ & $<0.001$ \\
miR-3613-3p & -0.84 & $-0.94 \sim-0.66$ & $<0.001$ \\
miR-4668-5p & -0.72 & $-0.88 \sim-0.46$ & $<0.001$ \\
miR-6750-5p & -0.37 & $-0.67 \sim-0.01$ & 0.04 \\
\hline
\end{tabular}

RT-qPCR, real-time quantitative polymerase chain reaction; $r$, Spearman correlation coefficient; $95 \% \mathrm{Cl}, 95 \%$ confidence interval. 
Table 5 (on next page)

Correlations between candidate miRNAs and clinical parameters in patients with IgA nephropathy.

$r$, Spearman correlation coefficient; $95 \% \mathrm{Cl}, 95 \%$ confidence interval; MAP, mean aortic pressure; UPE, 24-hour urinary protein excretion; Scr, serum creatinine; eGFR; estimated glomerular filtration rate. 
Table 5. Correlations between candidate miRNAs and clinical parameters in patients with IgA nephropathy.

\begin{tabular}{lccccccc}
\hline & \multicolumn{3}{c}{ miR-3613-3p } & & \multicolumn{3}{c}{ miR-4668-5p } \\
\cline { 2 - 5 } \cline { 6 - 8 } & $\mathrm{r}$ & $95 \% \mathrm{Cl}$ & $P$ value & & $\mathrm{r}$ & $95 \% \mathrm{Cl}$ & $P$ value \\
\hline Age & 0.12 & $-0.37 \sim 0.54$ & 0.631 & & 0.26 & $-0.28 \sim-0.67$ & 0.29 \\
MAP & 0.42 & $-0.06 \sim 0.72$ & 0.084 & & 0.34 & $-0.19 \sim-0.73$ & 0.173 \\
UPE & 0.501 & $0.03 \sim 0.83$ & 0.034 & & 0.44 & $-0.05 \sim-0.76$ & 0.066 \\
Scr & 0.451 & $-0.06 \sim 0.79$ & 0.06 & & 0.42 & $-0.06 \sim-0.73$ & 0.083 \\
eGFR & -0.48 & $-0.84 \sim 0.02$ & 0.043 & & -0.5 & $-0.84 \sim-0.08$ & 0.034 \\
Lee's grades & 0.57 & $0.07 \sim 0.84$ & 0.014 & & 0.57 & $0.04 \sim 0.85$ & 0.013 \\
\hline
\end{tabular}

$r$, Spearman correlation coefficient; $95 \% \mathrm{Cl}, 95 \%$ confidence interval; MAP, mean aortic pressure; UPE, 24-hour urinary protein excretion; Scr, serum creatinine; eGFR; estimated glomerular filtration rate. 